\title{
Study and Realization of a Prototype of a Drone with Eight Motors
}

\author{
Saidi Hemza ${ }^{1}$,Rachid Taleb ${ }^{2}$,Djebri Boualem ${ }^{3}$,Arhab Fatma ${ }^{4}$, Zemmour Mohammed $^{5}$ \\ ${ }^{1,5}$ Electrical Engineering Department, Mohamed Boudiaf University of Science and Technology,Oran, Algeria; \\ E-mail: h.saidi@univ-chlef.dz \\ ${ }^{2}$ Electrical Engineering Department, Hassiba Benbouali University Laboratoire Génie Electrique et Energies \\ Renouvelables (LGEER), Chlef, Algeria \\ ${ }^{3,4}$ Laboratory City, Architecture and HeritagePolyethnique school of architecture and Town planning (Algeria
}

Abstract-During this work we will attempt to give a presentation of a model, which implements the realization of an eight-engine drone in order to make it more stable. Indeed, it should be mentioned that the realization of a drone requires the mastery of various specialties such as: Mechanics, Aerodynamics, Control theory, Control and Computing. Throughout this article we have tried to mention the different models that we can find in the military or civil markets. By making a comparison of the two families (fixed wing and rotating wing) of the drones in order to choose the model to be realized, as a result of what we can notice.

Keyword - Drone, Aerodynamics, Control, BLDC motor.

\section{INTRODUCTION}

In order to design a flight controller, we must first deeply understand the movements of the drone, including its dynamics and its equations. Indeed, the comprehension of these latter is necessary for the design of the controller on one side and to ensure simulations of the air vehicle's behavior if they are closer than possible to reality on the other side.

The octocopter is characterized by its classification in the category of the most complex flying systems by referring to several factors such as: the number of physical effects, which affect its dynamics according to aerodynamic effects, gravity, gyroscopic effects, the friction and finally the moment of inertia.

\section{GENERAL DESCRIPTION OF THE OCTOCOPTER}

Using a modeling model of a quadrirotor, we have succeeded to produce a model of our octocopter. By dividing the force of a rotor on an arm of the quadrirotor on two symmetric forces similar in comparison with the arm of the quadrirotor. This, is done for each arm of the quadrirotor, then, it is enough just to manipulate the equations of the quadrirotor to obtain a model of our octocopter drone.

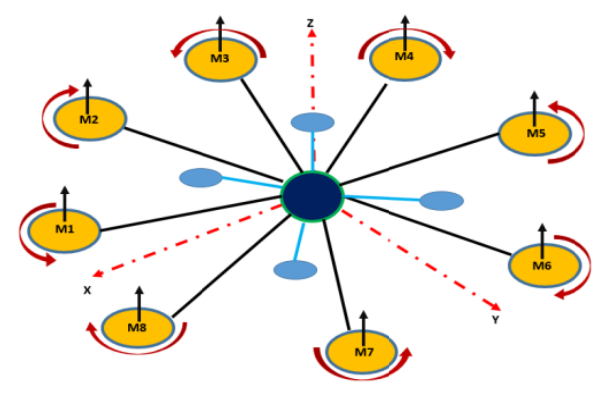

Fig 1:The basis of our modeling.

\section{DYNAMIC MODEL OF THE OCTOCOPTER}

The modeling of flying robots is a delicate task because the dynamics of the system are strongly nonlinear and fully coupled. In order to understand the dynamic model developed below, here are the various simplifying hypotheses on which we have based our work:

-The structure of the octocopter is assumed to be rigid and symmetric, which implies that the inertia matrix will be assumed to be diagonal.

The helices are supposed to be rigid, so that they can neglect the effect of their deformation during rotation.

The center of the mass coincides with the origin of the reference point linked to the structure.

The lift forces and the trains are proportional to the squares of the rotational speed of the rotors, which is an approximation very close to the aerodynamic behavior. 


\section{TRANSFORMATION MATRIX}

Any point in space can be defined by Cartesian, cylindrical or spherical coordinates. In general, the favorable system in our study is the Cartesian coordinate system which allows designating a rotation by the Euler angles.

To obtain the mathematical model representing the behavior of the octocopter,we use two reference points: one fixed reference point linked to the land (operator) $\mathrm{A}(\vec{X}, \vec{Y}, \vec{Z})$ and another movable $\mathrm{B}(\vec{x}, \vec{y}, \vec{Z})$ linked to the robot.

The advantage of the fixed reference mark of the operator is to evaluate the trajectory and the movement of the moving frame.

For the passage between the two landmarks the aeronautical community generally uses the rotation. first around the axis $\vec{z}$, then around the axis $\vec{y}$, et finally around the axis $\vec{x}$. These rotations are respectively expressed by three rotation matrices $\mathrm{R}(\vec{x}, \Phi), \mathrm{R}(\vec{y}, \theta)$ et $\mathrm{R}(\vec{z}, \Psi)$.

The passage of the fixed mark $\mathrm{A}(\vec{X}, \vec{Y}, \vec{Z})$ to the mark $\mathrm{B}(\vec{x}, \vec{y}, \vec{z})$ is made through the total rotation matrix $\mathrm{R}=\mathrm{R}(\vec{z}, \Psi) \cdot \mathrm{R}(\vec{y}, \theta) \cdot \mathrm{R}(\vec{x}, \Phi)$, such as:

$$
\begin{aligned}
& \mathrm{R}=\operatorname{Rot}_{z}(\Psi) \times \operatorname{Rot}_{y}(\theta) \times \operatorname{Rot}_{x}(\Phi)=\left[\begin{array}{lll}
c \Psi & -s \Psi & 0 \\
s \Psi & c \Psi & 0 \\
0 & 0 & 1
\end{array}\right] \times\left[\begin{array}{lll}
c \theta & 0 & s \theta \\
0 & 1 & 0 \\
-s \theta & 0 & c \theta
\end{array}\right] \times\left[\begin{array}{lcr}
1 & 0 & 0 \\
0 & c \Phi & -s \Phi \\
0 & s \Phi & c \Phi
\end{array}\right] \\
& \mathrm{R}=\left[\begin{array}{ccr}
c \Psi_{c} \theta & s \Phi s \theta c \Psi-s \Psi_{c} \Phi & c \Phi_{s} \theta c \Psi+s \Psi_{s} \Phi \\
s \Psi c \theta & s \Phi s \theta s \Psi+c \Psi_{c} \Phi & c \Phi_{s} \theta s \Psi-s{ }_{c} \Psi \\
-s \theta & s \Phi c \theta & c \Phi c \theta
\end{array}\right]
\end{aligned}
$$

With : $\mathrm{c}=\cos$, and $\mathrm{s}=\sin$

The rotational speeds $\Omega_{1}, \Omega_{2}, \Omega_{3}$ In the fixed frame are expressed as a function of the rotational speeds $\dot{\Phi}, \dot{\theta}, \dot{\Psi}$ by the following relation:

$\Omega=[\dot{\Phi} \dot{\theta} \dot{\Psi}]^{T}$

We note that the hypothesis of the motion in small angles is taken into account, that is to say that the octocopter makes small rotations, which allows to make the following approximations :

$\mathrm{c} \Phi=\mathrm{c} \theta=\mathrm{c} \Psi=1$, et $\mathrm{s} \Phi=\mathrm{s} \theta=\mathrm{s} \Psi=0$

For the translation speeds we have:

The linear speeds $v_{x}^{b}, v_{y}^{b}, v_{z}^{b}$, In the fixed frame according to the linear speeds $v_{x}^{m}, v_{y}^{m}, v_{z}^{m}$. In the mobile frame are given by:

$v=\left[\begin{array}{c}v_{x}^{b} \\ v_{y}^{b} \\ v_{z}^{b}\end{array}\right]=\mathrm{R} \times\left[\begin{array}{c}v_{x}^{m} \\ v_{y}^{m} \\ v_{z}^{m}\end{array}\right]$

\section{PHYSICAL EFFECTS ON OCTOCOPTER}

The physical effects that affect the octocopter are:

$A$. The weight of the octocopter

It is given by:

$\mathrm{P}=\mathrm{mg}$

whereas : $\mathrm{m}$ is the total mass and $\mathrm{g}$ the gravity.

$B$. The lift forces

These are forces which are caused by the rotation of the motors, they are perpendicular to the plane of the propellers. These forces are proportional to the square of the rotation's speed of the motors:

$F_{i}=b \omega_{i}^{2}$

With $i=\{1: 8\}$ is the index of the rotor, and $b$ is the lift coefficient $\left[\mathrm{kgm} / \mathrm{rad}^{2}\right]$, It depends on the shape of the blades and the density of the air.

\section{The drag force}

The drag force is the coupling between a pressure force and the viscous friction force, in this case there are two drag forces acting on the system:

The drag in the propellers: it acts on the blades, it is proportional to the square of the rotation's speed of the helix, it is given by the relation:

$T_{h}=d \omega_{i}^{2}$

$\mathrm{D}$ is the coefficient of drag which depends on the manufacture of the propeller.

D. Moments due to lift forces 
The translation on the x-axis: This is due to the moment created by the difference between the lift forces of the rotors $1,2,7,8$ and $3,4,5,6$

$$
M_{y}=l\left(F_{1}+F_{2}-F_{3}-F_{4}-F_{5}-F_{6}+F_{7}+F_{8}\right)=l b\left(\omega_{1}^{2}+\omega_{2}^{2}-\omega_{3}^{2}-\omega_{4}^{2}-\omega_{5}^{2}-\omega_{6}^{2}+\omega_{7}^{2}+\omega_{8}^{2}\right)
$$

$l$ is the length of the arm between the rotor and the center of gravity of the octocopter.

The translation on the $y$ axis is due to the moment created by the difference between the lift forces of the rotors $1,2,3,4$ and $5,6,7,8$. This moment is given by the following relation:

$M_{x}=l\left(F_{1}+F_{2}+F_{3}+F_{4}-F_{5}-F_{6}-F_{7}-F_{8}\right)=l b\left(\omega_{1}^{2}+\omega_{2}^{2}+\omega_{3}^{2}+\omega_{4}^{2}-\omega_{5}^{2}-\omega_{6}^{2}-\omega_{7}^{2}-\omega_{8}^{2}\right)$

E. Moment due to drag forces

- The rotation around the $\mathrm{z}$ axis: it is due to a reactive torque caused by the pairs trained in each helix, this moment is given by the following relation:

$M_{z}=d\left(\omega_{1}^{2}+\omega_{2}^{2}-\omega_{3}^{2}-\omega_{4}^{2}+\omega_{5}^{2}+\omega_{6}^{2}-\omega_{7}^{2}-\omega_{8}^{2}\right)$

F. Gyroscopic effect

The gyroscopic effect is defined as the difficulty of modifying the position or the orientation of the plane of rotation of a rotating mass. The gyroscopic effect is thus named in reference to the mode of operation of the gyroscope, a motion control apparatus used in aviation (from the Greek Györ which means rotation and scope, observe).

Indeed, rotating an object in rotation about a given axis creates a gyroscopic torque which is perpendicular to these two axes. The pivoting of the rotors with a certain angle gives rise to gyroscopic torques which are the vector product of the kinetic moments of the rotors and of the swing speed vectors.

They expressed themselves in the references related to the rotors by these relations:

During a rotation around $\mathrm{y}$, the gyroscopic torque on the $\mathrm{x}$ axis is:

$M_{x g}=J_{r o t} \Omega_{y}\left(\omega_{1}^{2}+\omega_{2}^{2}-\omega_{3}^{2}-\omega_{4}^{2}+\omega_{5}^{2}+\omega_{6}^{2}-\omega_{7}^{2}-\omega_{8}^{2}\right)$

Similarly, we obtain the gyroscopic torque on the y axis during a rotation around $\mathrm{y}$ :

$M_{y g}=J_{r o t} \Omega_{x}\left(-\omega_{1}^{2}-\omega_{2}^{2}+\omega_{3}^{2}+\omega_{4}^{2}-\omega_{5}^{2}-\omega_{6}^{2}+\omega_{7}^{2}+\omega_{8}^{2}\right)$

\section{DEVELOPMENT OF THE MATHEMATICAL MODEL ACCORDING TO NEWTON-EULER}

Based on the calculation of variation of the Euler-Lagrange system. Generalized coordinates determined the development of the rotational dynamics of the octocopter.

$\mathrm{q}=(\Phi, \theta, \Psi)$

Whereas $\Phi, \theta$ and $\Psi$ Are the Euler angles which represent the octocopter orientation in the moving frame B.

The difference between the kinetic energy $\mathrm{T}$ (caused by the angular velocities and the linear translation velocities) and the potential energy $\mathrm{V}$ denotes the Lagrangian :

$L=T-V$

The Euler-Lagrange equation is used to obtain the rotation dynamics of the octocopter. The kinetic energy of the octocopter is expressed by the following equation:

$T=\frac{1}{2} I_{x}(\Phi-\Psi \sin (\theta))^{2}+\frac{1}{2} I_{y}(\theta \cos (\Phi)+\Psi \sin (\Phi) \cos (\theta))^{2}+\frac{1}{2} I_{z}(\theta \sin (\Phi)-\Psi \cos (\Phi) \cos (\theta))^{2}$

The potential energy is expressed in the fixed reference $\mathrm{E}$ by:

$V=g \int x d m \cdot(-g \sin (\theta))+\int y d m \cdot(g \sin (\Phi) \cos (\theta))+\int z d m \cdot(g \cos (\Phi) \cos (\theta))$

The Euler-Lagrange equation becomes:

$\Gamma_{i}=\frac{d}{d t}\left(\frac{\partial L}{\partial q_{i}}\right)-\left(\frac{\partial L}{\partial q_{i}}\right)$

$\Gamma_{i}$ The extreme forces and $q_{i}(\mathrm{i}=\Phi, \theta, \Psi)$ The generalized coordinates.

For rolling:

$\frac{d}{d t}\left(\frac{\partial L}{\partial \Phi}\right)-\frac{\partial L}{\partial \Phi}=I_{x} \omega_{x}-\left(I_{y}-I_{z}\right) \omega_{y} \omega_{z}+\int y d m(y) \cdot(-g \cos \Phi \cos \theta)+\int z d m(z) \cdot(g \sin \Phi \cos \theta)$

For pitching:

$\frac{d}{d t}\left(\frac{\partial L}{\partial \theta}\right)-\frac{\partial L}{\partial \theta}=-\sin \Phi\left(\omega_{z} I_{z}-\omega_{x} \omega_{y}\left(I_{x}-I_{y}\right)\right)+\cos \Phi\left(\omega_{y} I_{y}-\omega_{x} \omega_{z}\left(I_{z}-I_{x}\right)\right)$

$$
+\int x d m(x) \cdot(-g \cos \theta)-\int y d m(y) \cdot(g \sin \Phi \sin \theta)-\int z d m(z) \cdot(-g \cos \Phi \sin \theta)
$$

And finally for the lace:

$\frac{d}{d t}\left(\frac{\partial L}{\partial \Psi}\right)-\frac{\partial L}{\partial \Psi}=-\sin \theta\left(\omega_{x} I_{x}-\omega_{y} \omega_{z}\left(I_{y}-I_{z}\right)\right)+\sin \Phi \cos \theta\left(\omega_{y} I_{y}-\omega_{x} \omega_{z}\left(I_{x}-I_{z}\right)\right)+\cos \Phi \cos \theta\left(\omega_{z} I_{z}-\right.$

$\left.\omega_{x} \omega_{y}\left(I_{x}-I_{y}\right)\right)$ 
Considering the hypothesis of small angles which the velocities of the angles of Euler $(\dot{\Phi}, \dot{\theta}, \dot{\Psi})$ are identical to the angular velocities in the movable reference frame $\left(\omega_{x}, \omega_{y}, \omega_{z}\right)$, the equations of motion become:

$$
\left\{\begin{array}{l}
\Gamma_{\Phi}=I_{x} \omega_{x}+\left(I_{z}-I_{y}\right) \omega_{y} \omega_{z} \\
\Gamma_{\theta}=I_{y} \omega_{y}+\left(I_{x}-I_{z}\right) \omega_{x} \omega_{z} \\
\Gamma_{\Psi}=I_{z} \omega_{z}+\left(I_{y}-I_{x}\right) \omega_{x} \omega_{y}
\end{array}\right.
$$

By equalization the equation's system (9) with (22) and by isolating the accelerations, we obtain:

$$
\left\{\begin{array}{c}
\ddot{\Phi} I_{x}=J_{\text {rotor }} \dot{\theta}\left(\omega_{1}^{2}+\omega_{2}^{2}+\omega_{5}^{2}+\omega_{6}^{2}-\omega_{3}^{2}-\omega_{4}^{2}-\omega_{7}^{2}-\omega_{8}^{2}\right)+\left(I_{y}-I_{z}\right) \dot{\theta} \dot{\Psi}+b l\left(\omega_{1}^{2}+\omega_{2}^{2}+\omega_{3}^{2}+\omega_{4}^{2}-\omega_{5}^{2}-\omega_{6}^{2}-\omega_{7}^{2}-\omega_{8}^{2}\right) \\
\ddot{\theta} I_{y}=J_{r o t o r} \dot{\Phi}\left(-\omega_{1}^{2}-\omega_{2}^{2}-\omega_{5}^{2}-\omega_{6}^{2}+\omega_{3}^{2}+\omega_{4}^{2}+\omega_{7}^{2}+\omega_{8}^{2}\right)+\left(I_{z}-I_{x}\right) \dot{\Phi} \dot{\Psi} \\
+b l\left(-\omega_{1}^{2}-\omega_{2}^{2}+\omega_{3}^{2}+\omega_{4}^{2}+\omega_{5}^{2}+\omega_{6}^{2}-\omega_{7}^{2}-\omega_{8}^{2}\right) \\
\ddot{\Psi} I_{z}=d\left(\omega_{1}^{2}+\omega_{2}^{2}-\omega_{3}^{2}-\omega_{4}^{2}+\omega_{5}^{2}+\omega_{6}^{2}-\omega_{7}^{2}-\omega_{8}^{2}\right)+\left(I_{x}-I_{y}\right) \dot{\theta} \dot{\Phi}
\end{array}\right.
$$

This model is based on the assumption of small angles.

\section{MODELING ACCORDING TO NEWTON-EULER}

The equation (22) is used to describe the dynamics of rotation, while the Newton-Euler equation is used to describe the dynamics of translation.

$\mathrm{m} \vec{a}=\vec{P}+\sum_{i} \vec{F}_{i}$

Avec $\vec{P}$ le poids, $\sum_{i} \vec{F}_{i}$ représente la somme des portances. Par l'utilisation de l'hypothèse de portance :

$\sum_{i} \vec{F}_{i}=b\left(\Omega_{1}^{2}+\Omega_{2}^{2}+\Omega_{3}^{2}+\Omega_{4}^{2}+\Omega_{5}^{2}+\Omega_{6}^{2}+\Omega_{7}^{2}+\Omega_{8}^{2}\right) \vec{z}=\sum_{i}\left(F_{i}, \vec{z}\right)$

Then we can write :

$\mathrm{m}\left(\begin{array}{c}\ddot{\mathrm{X}} \\ \ddot{Y} \\ \ddot{\mathrm{Z}}\end{array}\right)_{\left(\begin{array}{lll}\vec{X} & \vec{Y} & \vec{Z}\end{array}\right)}=m g\left(\begin{array}{c}0 \\ 0 \\ -1\end{array}\right)_{\left(\begin{array}{lll}\vec{X} & \vec{Y} & \vec{Z}\end{array}\right)}+\sum_{i} \vec{F}_{i}\left(\begin{array}{c}c(\Psi) s(\theta) c(\Phi)+s(\Psi) s(\Phi) \\ s(\Psi) c(\theta) c(\Phi)-c(\Psi) s(\Phi) \\ c(\theta) c(\Phi)\end{array}\right)_{\left(\begin{array}{lll}\vec{X} & \vec{Y} & \vec{Z}\end{array}\right)}$

This dynamic model is adopted by several authors

The translation model is obtained by developing the equation:

$\left\{\begin{array}{c}\ddot{\mathrm{X}}=\frac{(\cos \Psi \sin \theta \cos \Phi+\sin \Psi \sin \Phi)}{m} \sum_{i} F_{i} \\ \ddot{\mathrm{Y}}=\frac{(\sin \Psi \sin \theta \cos \Phi-\cos \Psi \sin \Phi)}{m} \sum_{i} F_{i} \\ \ddot{\mathrm{Z}}=-g+\frac{(\cos \theta \cos \Phi)}{m} \sum_{i} F_{i}\end{array}\right.$

The complete model according to Euler-Lagrange and Newton-Euler represents the dynamics of the octocopter is given by the following equations:

$$
\left\{\begin{array}{c}
\ddot{X}=\frac{(\cos \Psi \sin \theta \cos \Phi+\sin \Psi \sin \Phi)}{m} \sum_{i} F_{i} \\
\ddot{Y}=\frac{(\sin \Psi \sin \Phi \cos \theta-\cos \Psi \sin \Phi)}{m} \sum_{i} F_{i} \\
\ddot{Z}=\frac{\cos \theta \cos \Phi}{m} \sum_{i} F_{i} \\
\ddot{\Phi} I_{x}=J_{\text {rotor }} \dot{\theta}\left(\omega_{1}^{2}+\omega_{2}^{2}+\omega_{5}^{2}+\omega_{6}^{2}-\omega_{3}^{2}-\omega_{4}^{2}-\omega_{7}^{2}-\omega_{8}^{2}\right)+\left(I_{y}-I_{z}\right) \dot{\theta} \dot{\Psi}+b l\left(\omega_{1}^{2}+\omega_{2}^{2}+\omega_{3}^{2}+\omega_{4}^{2}-\omega_{5}^{2}-\omega_{6}^{2}-\omega_{7}^{2}-\omega_{8}^{2}\right) \\
\ddot{\theta} I_{y}=J_{\text {rotor }} \dot{\Phi}\left(-\omega_{1}^{2}-\omega_{2}^{2}-\omega_{5}^{2}-\omega_{6}^{2}+\omega_{3}^{2}+\omega_{4}^{2}+\omega_{7}^{2}+\omega_{8}^{2}\right)+\left(I_{z}-I_{x}\right) \dot{\Phi} \dot{\Psi} \\
+b l\left(-\omega_{1}^{2}-\omega_{2}^{2}+\omega_{3}^{2}+\omega_{4}^{2}+\omega_{5}^{2}+\omega_{6}^{2}-\omega_{7}^{2}-\omega_{8}^{2}\right) \\
\ddot{\Psi} I_{z}=d\left(\omega_{1}^{2}+\omega_{2}^{2}-\omega_{3}^{2}-\omega_{4}^{2}+\omega_{5}^{2}+\omega_{6}^{2}-\omega_{7}^{2}-\omega_{8}^{2}\right)+\left(I_{x}-I_{y}\right) \dot{\theta} \dot{\Phi}
\end{array}\right.
$$

We notice that the model presents strong nonlinearities and a great coupling between the control inputs, also between the angles because of the gyroscopic effects and between the dynamics of rotation and the dynamics of translation.

On pose : $\bar{\Omega}_{r}=-\omega_{1}^{2}-\omega_{2}^{2}+\omega_{3}^{2}+\omega_{4}^{2}+\omega_{5}^{2}+\omega_{6}^{2}-\omega_{7}^{2}-\omega_{8}^{2}$

Onsequently, the complete dynamic model which governs the octocopter is known according to the following equations. 


$$
\left\{\begin{array}{c}
\ddot{\Phi}=\frac{\left(I_{y}-I_{z}\right)}{I_{x}} \dot{\theta} \dot{\Psi}-\frac{J_{r}}{I_{x}} \bar{\Omega}_{r} \dot{\theta}+\frac{1}{I_{x}}\left(u_{5}+u_{6}\right) \\
\ddot{\theta}=\frac{\left(I_{z}-I_{x}\right)}{I_{y}} \dot{\Phi} \dot{\Psi}+\frac{J_{r}}{I_{y}} \bar{\Omega}_{r} \dot{\Phi}+\frac{1}{I_{y}}\left(u_{3}+u_{4}\right) \\
\ddot{\Psi}=\frac{\left(I_{x}-I_{y}\right)}{I_{z}} \dot{\Phi} \dot{\theta}+\frac{1}{I_{y}}\left(u_{7}+u_{8}\right) \\
\ddot{\mathrm{x}}=\frac{1}{m} u_{x}\left(u_{1}+u_{2}\right) \\
\ddot{\mathrm{y}}=\frac{1}{m} u_{y}\left(u_{1}+u_{2}\right) \\
\ddot{\mathrm{z}}=\frac{\cos (\Phi) \cos (\theta)}{m}\left(u_{1}+u_{2}\right)-g
\end{array}\right.
$$

white :

$\left\{\begin{array}{l}u_{x}=c \Phi_{c} \Psi_{s} \theta+s \Phi_{s} \Psi \\ u_{y}=c \Phi_{s} \theta s \Psi-s \Phi_{c} \Psi\end{array}\right.$

And:

$$
\left\{\begin{array}{c}
\ddot{\Phi}=\frac{\left(I_{y}-I_{z}\right)}{I_{x}} \dot{\theta} \dot{\Psi}-\frac{J_{r}}{I_{x}} \bar{\Omega}_{r} \dot{\theta}+\frac{1}{I_{x}}\left(u_{5}+u_{6}\right) \\
\ddot{\theta}=\frac{\left(I_{z}-I_{x}\right)}{I_{y}} \dot{\Phi} \dot{\Psi}+\frac{J_{r}}{I_{y}} \bar{\Omega}_{r} \dot{\Phi}+\frac{1}{I_{y}}\left(u_{3}+u_{4}\right) \\
\ddot{\Psi}=\frac{\left(I_{x}-I_{y}\right)}{I_{z}} \dot{\Phi} \dot{\theta}+\frac{1}{I_{y}}\left(u_{7}+u_{8}\right) \\
\ddot{\mathrm{x}}=\frac{1}{m} u_{x}\left(u_{1}+u_{2}\right) \\
\ddot{\mathrm{y}}=\frac{1}{m} u_{y}\left(u_{1}+u_{2}\right) \\
\ddot{\mathrm{z}}=\frac{\cos (\Phi) \cos (\theta)}{m}\left(u_{1}+u_{2}\right)-g
\end{array}\right.
$$

\section{THE PID CONTROLLER}

The PID controller, also called PID (proportional, integrator, derivative or proportional, integral, derivative), is a control system, allowing to perform a closed-loop control of an industrial system or "process". It is the most widely used regulator in the industry, and it allows adjusting a large number of physical quantities.

In our case we chose to implement this corrector for its robustness and simplicity. From the test / error method, we can know the adjustment coefficients (P-gain, I-gain and D-gain)

To apply our PID controller, we need to make a measurement on our system to calculate the error. This measurement must be done regularly at a certain sampling frequency.

For our order we give the following equation:

Command $=$ P-gain * error + I-gain * sum errors $+\mathrm{D}$-gain * (error - former error $)$

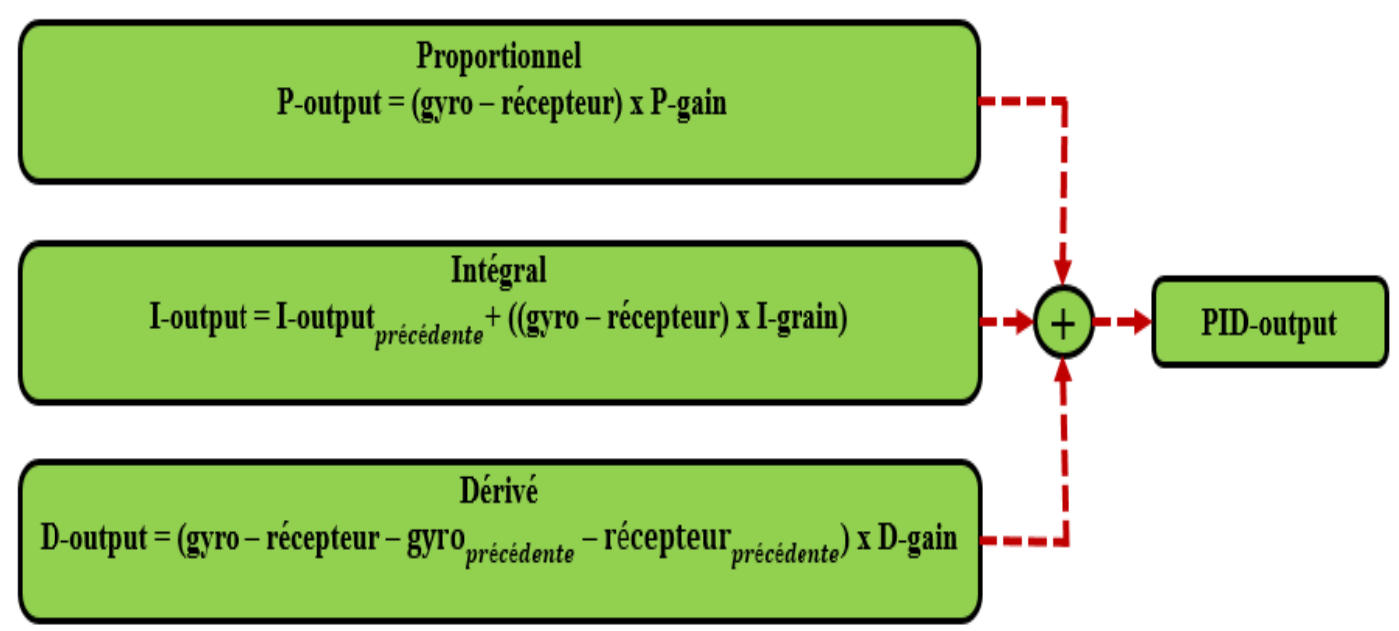

Fig 2:Descriptive diagram of the equations used in the PID 
To set the coefficients first, a simple proportional regulator must be set up (the coefficients I-gain and D-gain are therefore zero). By trial / error, the P-gain coefficient must be adjusted in order to improve the response time of the system. That is to say, a P-gain must be found which allows the system to get very close to the setpoint while being careful to keep the system stable: the system must not respond very quickly By oscillating much.

Once this coefficient is adjusted, we can pass to the coefficient I-gain. This one will make it possible to cancel the final error of the system. In order to the latter complies exactly with the set point. It is therefore necessary to set I-gain to have an exact response in a short time while trying to minimize the oscillations brought by the Integrator.

Finally, we can pass to the last coefficient D-gain which makes the system more stable. Its adjustment allows to reduce oscillations.

\section{DESCRIPTIVE DIAGRAM OF THE WIRING OF THE COMPONENTS ON THE OCTOCOPTER}

For our conception of an octocopter we will show how to choose the essential components for our realization with a description of each component. First of all, it is necessary to know the micro controller, the element that handles all the necessary data and calculations, then we evoke the elements assembled to the micro controller, for this we give a diagram which shows the management of the incoming and outgoing components of the microphone controller.

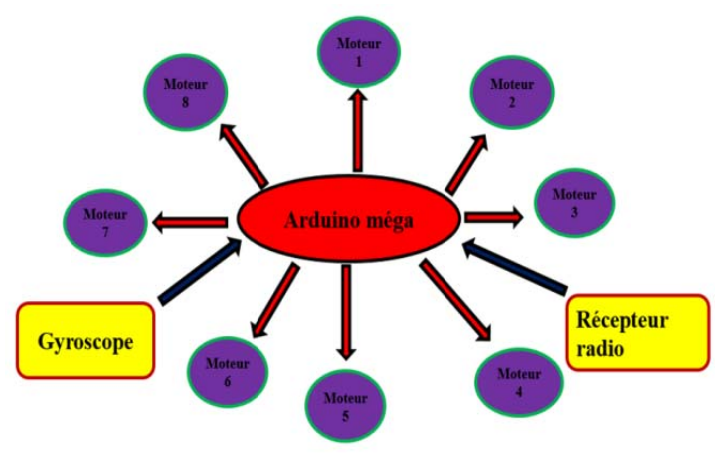

Fig 3:Diagram of the components. Personal treatment

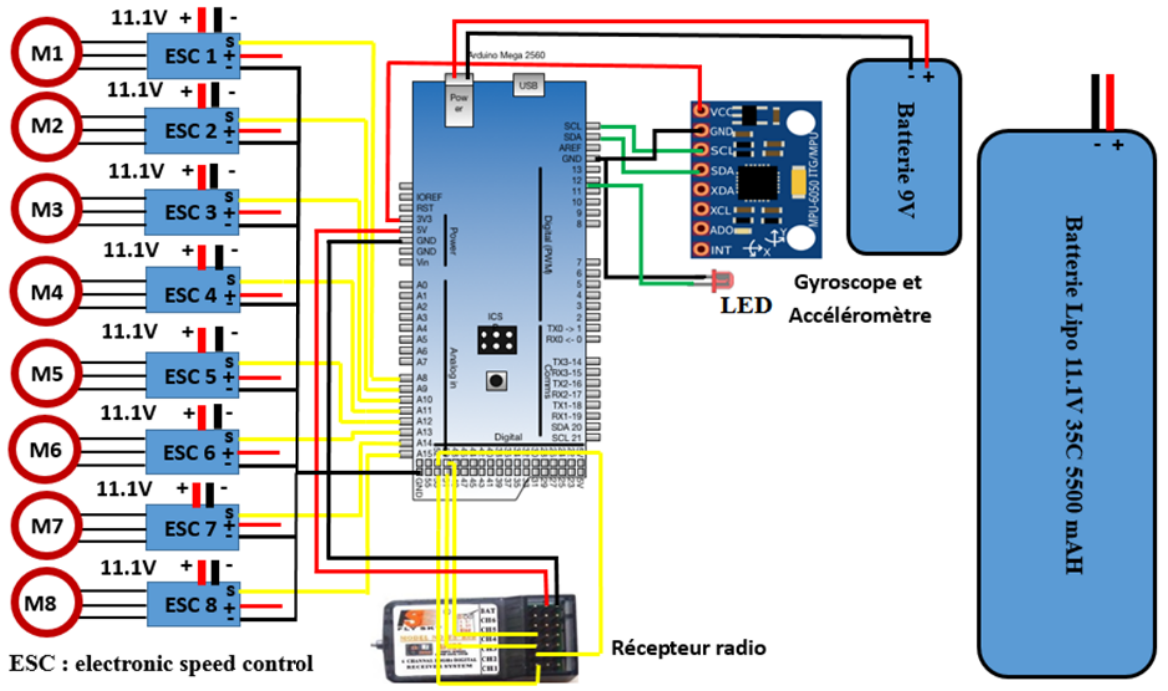

Fig 4:The electronic schema 
X ORGANIGRAM OF THE COMMAND

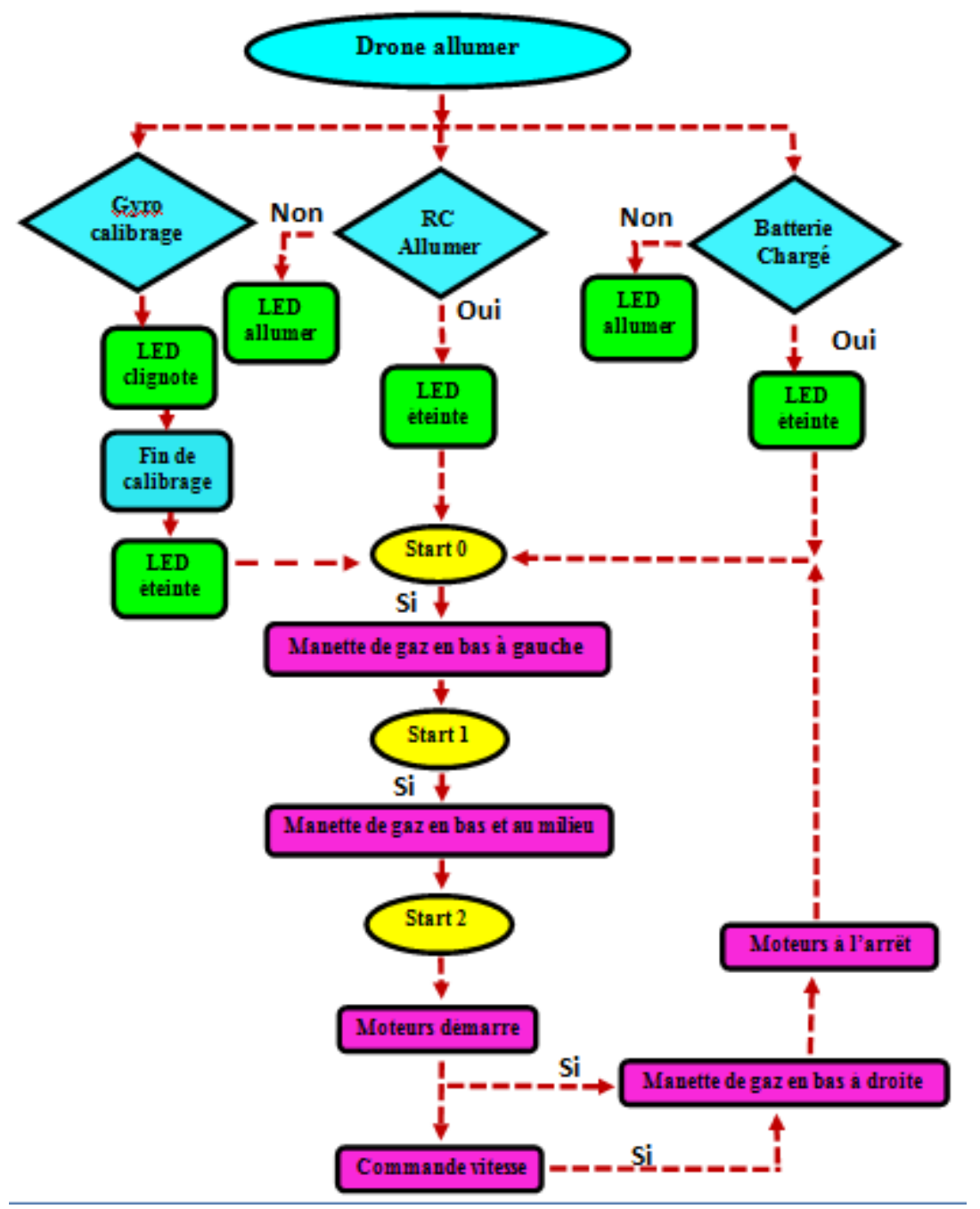

Fig5:Organigram of the command

\section{THE PROTOTYPE REALIZES}
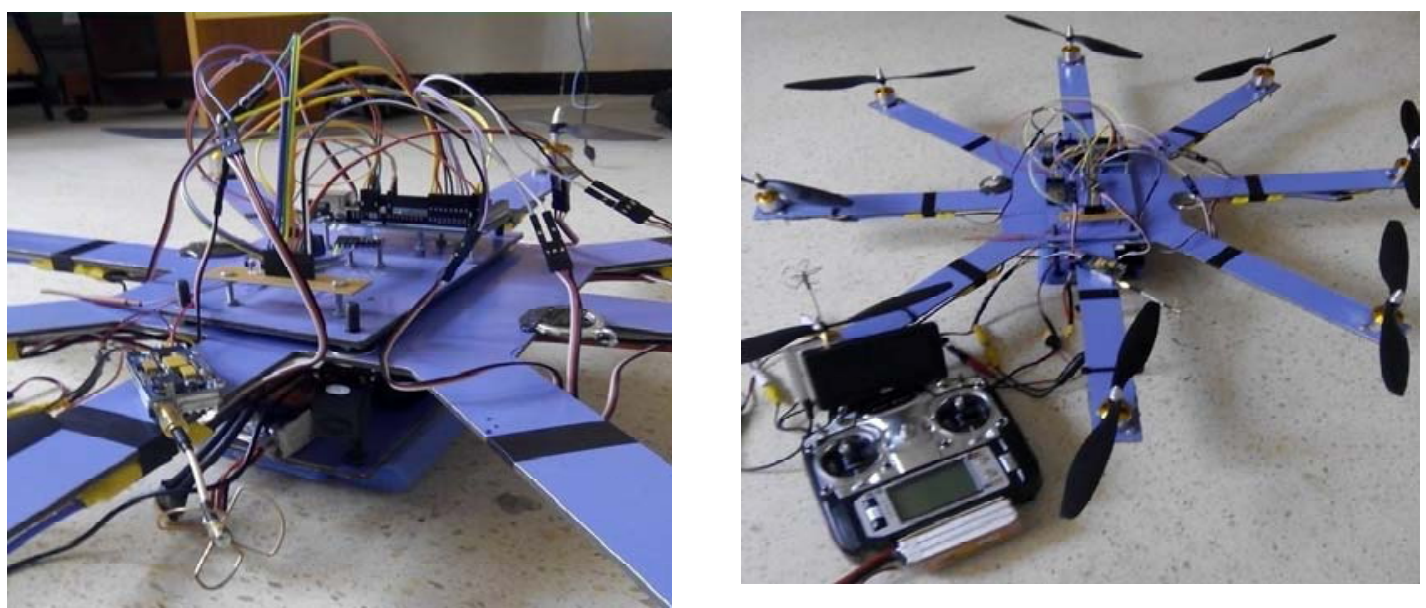

Fig 6: The realized prototype 


\section{CONCLUSION}

In order to become familiar with recent new technologies and with the growth of UAV interest, the growth of landing and takeoff airplanes (VTOL), The multorotors have known a great popularity in these last years and for the purpose of obtaining a degree in Electrical Engineering Master, we have chosen to accomplish a realization of a drone of eight engines to use it for " the agricultural fields such as spreading and fields's protection of diseases, despite the difficulties we have been facing as the less information needed to achieve our choice, we were able to accomplish our objective in realizing a drone of eight engines

First, we had to order the eight (8) engines of our drone, then we had met some problems during our project among them the non-synchronization of the drivers (ESC), for that we had to calibrate and modify our program according to the necessity for having a maximum synchronization. Despite this we always had a small margin of non-synchronization that could not be eliminated.

For getting good stability we have worked on the PID regulator (proportional, integral, derivative) with the test / error method which is long in its calculations but as it ensures both the errors made on the mechanical structure and the distribution of the weight, also the turbulent effects like the wind.

\section{REFERENCES}

[1] Book LES DRONES Functioning, Telepilotage, Applications, Reglimentation 2nd edition New regulation 2016 by Rodolphe Jobard.

[2] https://fr.wikipedia.org/wiki/Drone consulted in: 03/02/2017.

[3] Y.Zouaoui Boudjelthia and I.Settouti: Modeling and Control of a four-rotor in Six Degrees of Freedom. University hassiba benbouali of Chlef. Master's Thesis Promotion 2013.

[4] B.Musabyimana and B.Bouheraoua: Control of Systems by a $\mathrm{PI}^{\lambda} \mathrm{D}^{\mu}$ corrector of fractional order Application: Quadri-rotor control, Hassiba Benbouali University of Chlef. Master's Thesis, Promotion 2016.

[5] H.Khebache: Fault tolerance via the backstepping method of nonlinear systems Apllication: Quadrirotor type UAV system, University Ferhat Abbas de Setif. Magister's Thesis Promotion 2012.

[6] R.Ayad: Design and Construction of a Quadrirotor Drone, University of Science and Technology Mohamed Boudiaf d'Oran. Magister's Thesis, Promotion 2010. 